ment un certain but. Nous savons que, dans le colostrum, se trouvent, outre des antitoxines, des agglutinines qui quand elles passent avec la globuline dans le sang des jeunes animaux, les protègent contre beaucoup de maladies. Le lait de vache n'est certainement destiné qu'aux veaux, et il est par conséquent possible que les produits immunisants qui y sont contenus soient tout à fait sans importance pour les enfants qui, d'ailleurs, à l'encontre des veaux, déjà avant leur naissance reçoivent les immunisines de leur mère. Il me semble cependant qu'avant que la nature des agglutinines formatrices de crème soit plus amplement fixée, on doit se garder de chauffer le lait qui doit être employé à la nourriture des enfants tellement fortement que ces substances soient entièrement détruites. Jusqu'à nouvel ordre, la pasteurisation lente peut donc être consdiérée comme étant la meilleure méthode de pasteurisation basse du lait.

(A suivre)

\title{
LA PRODUCTION DU LAIT DE CONSOMIMATION EN DANEMARK
}

\author{
par J. de GIBON \\ Ingénieur Agronome \\ GÉNÉRALITÉS.
}

\section{Article Premier. - Le problème du latt a Copenhagne.}

Il ne saurait être question dans le cadre de cette étude d'étudier le problème du lait destiné à la consommation dans toute l'étendue du fays danois.

L'exemple du ravitaillement en lait à Copenhague sera seul envisa gé.

Les 730.000 habitants de Copenhague consomment journellement environ 275.000 litres de lait soit une moyenne de 4/10 de litre par habitant et par jour.

Un recensement de 1925 relève qu'en juillet de la même année, ce lait était fourni par 58.787 vaches appartenant à 3.610 étables.

Les 13.000 litres de lait pour enfants étaient produits par 2.000 vaches (19 étables) et les 3.500 litres de lait de Jersey par 1.000 vaches environ (10 étables).

$85 \%$ du lait venàu à Copenhague était soumis à un contrôle qualitatif. Ce dernier lajt était fourni en grande majorité par les cultivateurs fournisseurs des grandes laiteries, le reste par les laiteries coopératives de la campagne, dans la proportion de $85 \%$ à $15 \%$.

Les producteurs de lait se sont réunis en sociétés locales, fédérées elles-mêmes en un organisme central, pour la défense de leurs intérêts. lait.

3 grandes laiteries assurent à elles seules $85 \%$ du ravitaillement en

Ce sont deux sociétés privées, sociétés par actions : 
$1^{\circ}$ L'allianse Danske Maelke Compagni et Kjobenkavns Maelkeforsyning;

\section{$2^{\circ}$ L'Enigheden (l'Unité);}

$3^{0}$ et une société coopérative de grands propriétaires Trifolium (88 vaches par propriétaire adhérent en moyenne).

On trouvera plus loin des paragraphes consacrés à ces deux premières sociétés.

Lo lait nécessaire à la capitale se trouve facilement et en toutes saisons, grâse à l'alimentation rationnelle qui permet une production laitière constante pendant toute l'année.

2 modes de transport sont employés, à proportions sensiblement égales : le chemin de fer (plus coûteux) et le camion qui semble de plus en plus employé pour les distances moyennes.

Les distances des centres de production ont tendance à croître d'année en annés. Actuellement la répartition se fait comme il suit :

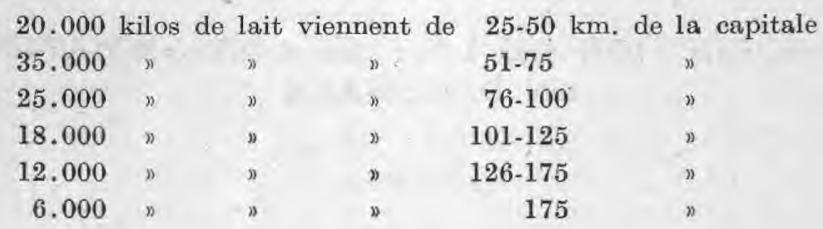

Il y a peu de trains de lait spéciaux; le plus souvent on accroche les wagons à des trains de denrées extrêmement rapides. La durée maxima du transport est de 5 heures.

$L_{x}$ vente du lait en ville est effectuée dans les laiteries de quartier ou par les voitures de livraison des soziétés : voitures à type allongé bien connu, sortes de " boutiques ambulantes " qui livrent le lait aux clients réguliers, comme aux clients de passage.

1.600 laiteries de quartier et voitures de livraison ont été recensées en 1925 par les soins de la Commission Sanitaire. Les crémeries achètent leur lait aux grandes sosiétés ou aux laiteries de campagne et vendent le lait en bouteilles ou à la mesure.

Les voitures de livraison ne vendent que du lait en bouteilles; la vente à la mesure étant seulement autorisée dans les laiteries de quartier.

Las laiteries de campagne et les cultivateurs qui font une concurrenze sérieuse aux grandes sociétés (prix de vente inférieur du lait, par suite des frais généraux sensiblement moins élevés), s'adressent aux détaillants ou fondent des dépôts en ville, car ils ne peuvent effectuer eux-mêmes la livraison directe ou la vente dite "à la voiture "; une semblable vente rendrait en effet tout contrôle impossible.

Paiement du lait. - Un Comitéde fixation du prix du lait à Copenhague, comité de 5 membres : 2 représentants des producteurs de lait (l'un nommé par les laiteries coopératives, l'autre par le reste des fournisseurs de lait), 2 représentants des laiteries de Copenhague, et le directeur 
du ravitaillement en denrées périssables de la capitale, président du Comité et représentant des consommateurs, fixe par semaine le prix du Jait.

Ce prix a pour base d'évaluation l'addition de 3 chiffres :

$1^{0} \mathrm{Le}$, cours hebdomadaire du beurre (cotation de Copenhague), élément donnant la valeur de la matière grasse ;

$2^{\circ}$ Le cours du lait écrémé, cours donné par le Comité de cotation des sociétés de laiteries;

$3^{\circ}$ Le montant des frais de transport.

Cette addition détermine le prix du lait rendu "gare Copenhague ».

Le cours du lait est déterminé pour un lait moyen à $3,5 \%$ de matière grasse.

Jusqu'à concurrence de $3,75 \%$ de matière grasse, il est donné une prime à la matière grasse. Cette prime a été baissée par suite de l'augmentation trop sensible qui en résultait pour les prix de vente au détail, et pour éviter des complications dans les crémeries.

Da plus, pour ce qui concerne le lait contrôlé, soit $85 \%$ du lait vendu à Copenhague, un ascord entre producteurs et marchands a abouti à la classification suivante, en qualités et en cours de ce lait.

Le résultat des épreuves de la réductase et du lacto-fermentateur détermine les 4 classes suivantes :

$1^{\text {re }}$ classe : prix fixé par le Comité : prix officiel.

$2^{\text {e }}$ classe : le prix officiel moins $1 / 2$ öre (env. 3 cent. 5 ).

$3^{\mathrm{e}}$ classe : le prix officiel moins 1 öre $1 / 2$ (env. 10 cent.).

$4^{\mathrm{e}}$ classe : lait impropre, renvoyé sans paiement au producteur.

En février 1928, le cours du lait était de 20 öres (soit 1 fr. 40, en 1928) le kilo, ce qui ramené au litre (base de la vente au détail) donne un prix de 21 öres $1 / 2$ soit : $1 \mathrm{fr}$. 50 environ le litre.

Le prix de vente au détail à la même époque est de 35 öres le litre pour le lait vendu en bouteilles, soit $2 \mathrm{fr}$. 45 environ.

Cette différence, soit 13 öres 1/2 (95 cent.) est assez élevée, bien qu'elle ait sensiblement diminué si l'on se reporte à une époque récente (juin 1927) à un exemple cité dans le livre de M. Coche (La Production laitière en Danemark) où la différence s'élevait à $1 \mathrm{fr} .20$ (2 fr. 25-1 fr. 05).

Cette somme représente les frais de traitement dans les laiteries, traitement minitieux qui entraîne des frais assez élevés.

Art. 2. - RÉglementation Et contrôle des produits lattiers.

\section{RÉGLEMENTATION :}

La réglementation visant le commerce des produits laitiers est édictée soit par des lois ou des ordonnances applicables à toute l'étendue du pays, soit par des arrêtés municipaux applicables dans les villes respectives.

\section{Ordonnances.}

La plus complète et la plus récente est l'ordonnance du Ministre de la Justice, qui remonte au 22 octobre 1925. Cette ordonnance déter. 
mins les dénominations et les définitions des différentes variétés de laits et de crèmes.

On pəut en résumer comme il suit les dispositions essentielles.

A. Laits : Quand le lait est vendu sans appellation spécifique, il est considéré comme lait doux, c'est-à-dire lait complet et frais. On ne peut mélanger à ce lait ni eau, ni lait écrémé pasteurisé ou chauffé. Ce lait doux doit avoir une teneur minima de $3 \%$ de matière grasse.

Toutes les autres catégories de laits ou crèmes doivent être livrées à la consommation sous les désignations suivantes correspondant à leur nature.

a) Lait de Jersey ou Jerseymaelk, lait provenant de vaches de la race de Jersey, contenant un minimum de $4,75 \%$ de matière grasse.

b) Lait écrémé, lait auquel on a enlevé une partie de sa matière grasse. Ce lait doit remplir les conditions signalées pour le lait frais complet. Si le lait contient au moins $0,75 \%$ de matière grasse, sa dénomination devient "lait $1 / 2$ écrémé ».

c) Le lait pour enfants "Bornemaelk ": c'est un lait frais, produit par des vaches tuberculinisées depuis moins d'un an et soumises à un contrôle régulier. Des conditions spécifiées par des arrêtés locaux sont déterminées en ce qui concerne la production et le traitement de ce lait.

d) Le lait dit "stérilisé " doit être venda dans des récipients dont la " fermature soit telle qu'elle permette une conservation parfaite du produit ".

e) Le Babeurre (Kaernemaelk), doit révéler à l'analyse une teneur minima de $6,5 \%$ d'extrait sec.

B. Crèmes : La crème doit être vendue sans addition d'eau, et dépourvue de tout eorps étranger.

La crème doit avoir une teneur minima de $9 \%$ de matière grasse naturelle. L'ordonnanse distingue 4 dénominations des crèmes suivant leur teneur en matière grasse :

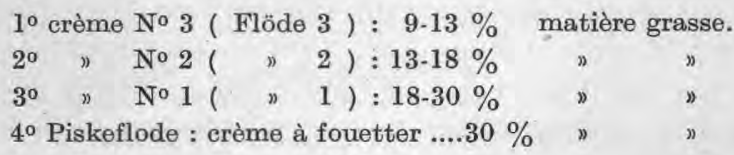

Pour la vente des crèmes en bouteilles, l'inscription de la teneur en matière grasse est obligatoire sur la capsule de fermeture.

C. Laits et crèmes : Ne peut être vendu comme lait ou crème "pasteurisée " que le produit qui a subi, au plus tard 24 heures a près la traite, un chauffage à $80^{\circ}$, suivi d'un refroidissement à $12^{\circ}$. De plus le lait pasteurisé doit être neutre à la réaction de Storch (paraphénylènediamine).

Los récipients des laits et crèmes pasteurisés doivent indiquer la date et le lieu de la pasteurisation. 
Interdiction est faite de mélanger lait pasteurisé et lait cru.

Tout autre procédé de chauffage pour ees produits nécessite, exception faite pour le lait condensé ou séché, une autorisation spéciale du Ministère de la Justice.

\section{$2^{\circ}$ Arrêtés municipaux.}

Certaines grandes villes ont parfois édicté par des arrêtés municipaux applicables dans ces communes, des dispositions encore plus strictes que celles contenues ci-dessus.

Copenhague, entre autres, a par un arrêté du 6 octobre 1924, venant après l'arrêté du 4 novembre 1918, rendu obligatoires pour le commerce du lait dans la capitale les dispositions qui suivent :

Le lait doit avoir une teneur minima de $3,25 \%$ de matière grasse et la crème će $13 \%$ (au lieu de $3 \%$ et $9 \%$ ).

Le lait est dit " pasteurisé » quand il a été chauffé au moins à 85 degrés et refroidi à moins de $10^{\circ}$ (au lieu de $80^{\circ}$ et $10^{\circ}$ ).

Une couleur anormale, comme une odeur et un goût pas naturels rendent un lait impropre à la consommation, donc à la vente. Il en va de même pour un lait malpropre (lait donnant un dépôt visible après deux heures de repos). Il est interait de conserver ou de colorer le lait par un prodnit quelconque.

La "Commission Sanitaire de Copenhague » a édicté, en une annexe jointe à l'arrêté ci-dessus les mesures concernant :

I. - La production du lait normal livré à la consommation dans Copenhague.

Des vaches atteintes de maladies comme la tuberculose ou la fièvre aphteuse, maladies révélées par un examen extérieur de l'animal, ne peuvent fournir du lait pour Copenhague.

Les contrôleurs officiels sont en droit d'exiger des laitiers des certificats de vétérinaire relatifs à l'hygiène et à l'alimentation des vaches fournissant le lait pour la vente.

Le producteur doit:

$1^{0}$ Faire inspecter son troupeau au moins mensuellement par un vétérinaire qui lui délivre un certificat dit " de- visite »;

$2^{\circ}$ Eloigner de l'étable ou séparer du troupeau suivant nécessité, dans le cas de maladies empêchant la vente à Copenhague du lait des vaches atteintes. Une autorisation du vétérinaire devra être attendue pour livrer le lait après guérison.

La traite de ces vaches sera effectuée à part de celle du reste du troupeau.

Un ensemble de dispositions concernant l'hygiène des animaux à l'étable et les soins à donner à la traite, est également énoncé.

II. - Boernemaelk ou lait pour enfants. 
Lo laitièr qui vend ce lait doit en faire connaître la provenance à la commission d'hygiène.

Quant au producteur, en plus des dispositions énoncées ci-dessus, il doit : soumettre ses vaches tous les ans à l'épreuve de la tuberculine, recevoir 2 fois par mois la visite du vétérinaire d'Etat, lui soumettre la ration de ses animaux (au plus 30 kilos par jour et par vache de racines ou tubercules, un kilo d'aliments mélassés, 500 gr. de tourteau de coton, interdiction des feuilles et pulpes de betteraves et de fourrages ensilés).

La traite ne paut être faite qu'à la main, avec des soins extrêmes de propreté. Aussitôt après la traite, on doit refroidir le lait à moins de $8^{\circ}$.

On a pris soin de "placarder" ces règles dans les quelques fermes spécialisées dans la production de ce lait.

Désinfection, lavage des étables, propreté méticuleuse du personnel, tout est mis en cuvre pour assurer la production et la récolte d'un lait aussi pur que possible.

Ce même soin est observé pour le traitement de ce lait dans les grandes laiteries de Copenhague, en ce qui concerne le nettoyage et la mise en bouteilles.

Une variété de ee lait, l'Ismaelk ou lait glacé, est réservée aux enfants délicats : la traite s'opère dans un local spécial, où bête par bête, les vaches viennent une fois nettoyées se faire traire. Le lait est refroidi à $0^{\circ}$.

Ls prix de revient de ce lait est sensiblement plus élevé, par suite des dépenses nécessitées par des soins semblables. En février 1928, il était payé au producteur 23 öres le litre ( $1 \mathrm{fr}$. 55), au lieu de 20 öres pour le lait ordinaire, soit 3 öres de plus (un peu plus de 20 centimes de différence).

Son prix de vente à la même époque est de 44 öres (soit $3 \mathrm{fr}$.) au lieu de 35 öres pour le lait naturel ( 2 fr. 40 ), soit un excédent de 60 centimes.

$3^{\circ}$ Réglementation des locaux de vente.

Ces dispositions sont relatives à la tenue des locaux de vente, à l'hygiène du personnel et à la propreté du matériel.

Aucun autre produit, excepté le pain, le beurre, les œufs, et la farine, ne psut être vendu dans le même local.

La pərsonnel de ces magasins de vente doit être surveillé, en ce qui est ralatif à l'état de santé : il doit être exempt de maladies contagieuses.

Une attention toute particulière doit veiller au nettoyage et à la stérilisation des récipients.

\section{Le contrôle :}

Ce contrôle est effectué dans la capitale par la Commission Sanitaire, service annexe de la Municipalité créé par le Ministère de l'Intérieur.

Le Directeur de la Police est président de la Commission qui reste toujours sous la dépendance du même Ministère. Un bourgmestre, 2 conseillers municipaux et le Médecin en Chef de la ville en sont mombres. 
Un pærsonnel de 12 médecins et de 3 vétérinaires exerce effectivement le contrôle, aidé par des vétérinaires de provinces (110 en 1925) pour les tournées " curales " et les inspecteurs de la police sanitaire pour les tournées dans la capitale.

L'inspection mensuelle revient aux vétérinaires locaux. Les visites dites exceptionnelles reviennent aux vétérinaires de la commission.

Les deux mesures de répression pour infraction consistent dans l'interdiction de vendre du lait à Copenhague, interdiction prononcée par la commission et dans les amendes fixées par les tribunaux compétents.

Ces sanctions d'interdiction de vente se sont montées à 11 en 1925 ; la même année il y eu 4 assignations en justice.

Des inspections des locaux de vente, avec prises d'échantillons pour Ia recherche de la M.G., de la teneur en eau, de l'état de propreté, complètent le çontrôle.

Le tableau suivant résume l'activité au cours de 1925 de ce service de contrôle.

10 Chez le producteur :

620 visites des vétérinaires de la commission

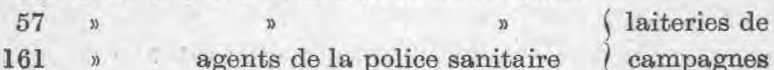

$2^{\circ}$ Chez le commerçant:

1.960 inspections de locaux de vente

281 voitures de livraisons laiteries

7.280 prises et analyses d'échantillons

donnant lieu à 484 cas de poursuite en comptant les avertissements simples.

Une réglementation et un contrôle du même type que celui précédemment exposé, existent dans d'autres agglomérations importantes. Le Ministère de l'Intérieur a du reste pris l'initiative de créer un modèle d'arrêté qui sert de fondement aux divers arrêtés locaux.

A ce contrôle officiel, il faut ajouter le contrôle privé exercé par les laiteries chez les producteurs en ce qui concerne la qualite du lait. Ce contrôle sur accord entre les agriculteurs intéressés et les grandes sociétés laitières, revient à la commission de Copenhague qui fixe le prix du lait.

\section{Art. 3. - L'approvisionnement de Copenhagun en latt.}

On se souvient des efforts considérables tentés de différentes manières, sur les conseils des hygiénistes spécialisés dans les questions d'alimentation. Depuis quelques années, ces efforts se sont surtout concentrés sur la question primordiale du lait, l'aliment par excellence pour tous, grands et petits.

Les grandes villes, émues par ces campagnes nombreuses en faveur 
d'un lait sain, ont pour la plupart tenté des essais plus ou moins fructueux pour la fourniture d'un lait dans les meilleures conditions possibles.

Le Danemark, qui depuis près de 50 ans a orienté tout spécialement sa production agricole vers la production laitière, constitue un pays où le commerce du lait se fait avec le maximum de garanties pour le consommateur.

Autrefois, des vaches nourries parcimonieusement, souvent malades, donnaient un lait que l'on récoltait sans soin, et que l'on conservait encore plus mal.

Le commerce du lait, que l'on omettait de surveiller, laissait la porte libre à toute une série de manœuvres frauduleuses, et donnait libre cours à des plaintes continuelles de la part des consommateurs.

Il n'en va plus de même maintenant. Une alimentation rationnelle, eonjuguée à des soins hygiéniques très développés vis-à-vis des animaux, a permis aux cultivateurs de fournir un lait parfait. Un contrôle très sévère de la part de l'Etat, comme de la part des grandes sociétés laitières empêche désormais les fraudes. La qualité du lait s'est considérablement accrue depuis les premières années de production laitière, et ce fait a amené une consommation croissante de cet aliment dans les villes. Les statistiques révèlent une consommation journalière de 0 litre 4 par habitant.

Copenhague qui groupe 700.000 âmes environ, absorbe quotidiennement 275.000 litres de lait.

Ce lait était jadis fourni par un grand nombre de laiteries : 200 environ. Ce nombre a considérablement diminué, et la fourniture du lait pour la capitale reste maintenant entre les mains de trois grandes laiteries, qui à elles seules fournissent $85 \%$ du lait absorbé.

\section{$1^{\circ}$ La Kjobenhavns Maelkeforsyning.}

La Kjobenhavns Maelkeforsyning est alliée maintenant à la Danske Maelke Compagni, une ancienne société rivale.

La K.M. est de création ancienne : inaugurée en 1878, elle apparaît à ses débuts comme une œuvre philanthropique ; un intérêt de $5 \%$ rémunérait les eapitaux engagés et le surplus des bénéfices constituait une caisse de réserve pour les agrandissements, les améliorations et surtout des distributions de lait à prix réduits ou gratuites aux indigents. Cette société a pris maintenant un caractère commercial.

Elle a joué un rôle prépondérant dans la transformation des conditions de la vente du lait.

A quelques centaines de mètres de la précédente, et à l'extrémité d'une rue portant un nom bien significatif "Maelkeveg" (le chemin du lait), la D. M. C. traitait une plus grande quantité de lait que sa rivale : 80.000 litres au lieu de 30.000 litres. Etablie sur les mêmes principes, à la fin du siècle dernier, ces deux sociétés jadis rivales ont vite compris 
l'intérêt économique qu'elles auraient à s'allier. C'est ce qu'elles ne tardèrent pas à faire dès 1916 à leur grand profit comme à celui des consommateurs. Une allianze sur des bases solides permettrait de donner une plus grande intensité aux efforts effectués pour améliorer le lait de consommation. K. M. fournit le lait directement aux consommateurs, D. M. C. aux grossistes, hôpitaux...

Actuellement, la grande 'Société traite 130.000 litres de lait par jour; elle alimente done plus du $1 / 3$ de la capitale.

Pour sa part, la K. M. traite dans ses bâtiments, de construction rérente (1925), près de 50.000 litres de lait. L'installation de la K. M. étant plus moderne encore s'il est possible que celle de la D. M. C., on trouvera ci-dessous, l'étude de l'installation et du fonctionnement de la première société.

La qualité d'un lait dépend de deux facteurs : $1^{\circ}$ un facteur de pro. duction : ce sont les conditions dans lesquelles il est recueilli; $2^{\circ}$ un facteur de traitements : ce sont les conditions dans lesquelles il est manseuvré à la laiterie.

Les 50.000 litres de lait traités journellement à la K. M. sont fournis par 35 grands fermiers. L'un d'eux fournit à lui seul 5.000 litres par jour. Cette concentration des producteurs facilite beaucoup la surveillance des étables produisant le lait envoyé à la K. M. C'est qu'en effet, ces étables sont soumises à un contrôle périodique effectué par des vétérinaires dépəndant de la société. Les vaches produisant le lait des enfants sont soumises à une visite bimensuelle, les autres à une visite mensuelle.

De plus, les vétérinaires du service de contrôle de l'Etat ont droit d'accès et d'inspection dans ces étables.

Toutes les vaches sont passées à la tuberculine; cette opération rendue obligatoire par la société, pour les vaches fournissant le lait à la laiterie, ne l'est pas aux yeux du législateur danois ; toutefois, elle est courante d'autant qu'elle se pratique gratuitement pour les cultivateurs, et qu'elle est effectuée aux frais de l'Etat par les vétérinaires officiels.

La so iété a édicté toute une série de règles concernant l'alimentation des vaches. Cette alimentation est suivie de près et contrôlée par les contrôleurs de la société. Certains aliments, comme ceux donnant du goût au lait, sont interdits ; la liste des tourteaux autorisés est dressée minutieusement.

Do plus, des conseils concernant les soins à apporter à la traite sont prodigués constamment (1).

Par contrat, les fournisseurs de la société s'engagent à refroidir le lait à $4^{\circ}$ aussitôt a près la traite.

Dans ce but, des facilités sont données aux agriculteurs pour obtenir les réfrigérants LAURENOE nécessaires à ce refroidissement; les appareils

(1) Le lait de mauvaise qualité est toujours renvoyé au producteur. 
leur sont ou prêtés moyennant une faibje indemnité (sorte de location), ou vendus à des prix très avantageux. La glace leur est procurée dans des conditions très modestes, et il doit en être fait un emploi d'au moins 40 kilos par 100 kilos de lait.

Cette refrigération, facile en hiver, pendant le séjour des vaches à l'étable, devient plus difficile en été, pendant le séjour aux pâturages ; des abris démontables sont alors fixés dans les champs et permettent d'abriter le réfrigérant à proximité du lieu de traite.

Cette opération permet au lait d'arriver à l'usine encore froid à $10^{\circ}$.

Lo nouveau bâtiment de la société, construit en 1925, s'étená sur $65 \mathrm{~m}$. de long avec $19 \mathrm{~m}$. de large. Il se compose de deux parties, l'une de 32 mètres de long à 3 étages respectifs de $4 \mathrm{~m}$. $50,5 \mathrm{~m} .50$ et $7 \mathrm{~m} .25$, et l'autre à deux étages de $4 \mathrm{~m} .50$ et $5 \mathrm{~m} .50$ qui sont reliées par un hall de 8 mètres de haut. Une tour ou château d'eau de $23 \mathrm{~m}$. 50 surplombe l'ensemble de la construction.

Ces bâtiments sont un véritable "modèle" de construction dans le genre :

Rien n’a été négligé, et tout jusqu'au plus petit détail a été conçu pour donner des salles hautes, spacieuses, aérées, éclairées, et d'une propreté exceptionnelle.

Las murs sont carrelés intérieurement en faïence ou porcelaine; le sol est pavé en potits carreaux de grés ou en asphalte. Le nettoyage et l'évacuation des eaux sont méthodiquement conçus. Pas d'angles aux murs, mais des coins arrondis et incurvés, où la poussière ne peut se loger. Sous chaque coussinet d'arbre de transmission une gouttière en métal recueille l'huile qui pourrait s'échapper. Toutes les tuyauteries sont recouvertes d'un cordon épais d'étoffe, fréquemment passé à la peinture blanche.

L'installation a été effectuée de manière à ce qu'il ne soit pas nécessaire de pompər le lait ou la crème; on ne trouve pas une pompe à lait. Celui-ci coule naturellement par différences de niveau d'un endroit a l'autre ; la Sosiété attache une grande importance à ce fait de ne pas faire pomper le lait: un envoi brutal du lait par pression nuit toujours à la qualité de celui-ei.

La plus grande partie du lait arrive par voie ferrée. La distance la plus éloignée est de 145 kilomètres. Il faut compter au plus 4 heures pour le transport. Les bidons arrivent dans des wagons spéciaux : Maelkevogn, analogues à nos wagons blanes français (wagons frigorifiques).

Le lait est contenu dans des bidons de 50 litres de forme courante et faits tous sur le même modèle. Ces récipients sont hermétiquement fermés et alignés côte à côte dans les "Maelkevogn ". Chaque bidon porte une étiquette indiquant le nom et l'adresse du producteur ainsi que la gare expéditrice.

Pour les transports les plus longs, l'expédition était faite autrefois 
dans des bidons d'une contenance de 500 litres : bidons de $1 \mathrm{~m}$. 50 de longueur environ et de $70 \mathrm{~cm}$. de diamètre. On renonce de plus en plus à ce procédé qui n'est plus guère em.ployé, car il était sujet à de ncmbreux inconvénients. Ce transport était employé pour des laits conservés à l'aide du prozédé de congélation, connu sous le nom de procédé CASSE qui devait assurer au lait un refroidissement parfait pendant la durée du transport: le dégel « du lait était délicat et un lait dégelé brutalement " subit toujours une altération partielle qu'on peut déceler au moment de la consommation.

Une voie ferrée arrive au pied du bâtiment côté Sud-Est; un autre quai voisin permet le déchargement des camions, ce qui assure le déchargemerit simultané des bidons arrivant par camions ou par voie ferrée. 80 à $85 \%$ des laits arrivent du reste par chemin de fer, et les camions ne font que ramasser le lait dans les centres de production immédiatement proches de la capitale.

Un tapis roulant transporte automatiquement les bidons au fur et à mesure des arrivages vers un monte-charge qui assure l'ascension des laits jusqu'au troisième étage, où une plate-forme dominant la salle des premiers traitements (réception, échantillonnage et pasteurisation) les reçoit.

Cette salle, qui est typique comme aménagement, est vaste : elle prend une hauteur de 7 mètres 25 et mesure toute la largeur du bâtiment.

La rézeption se fait lait par lait, suivant l'origine de production, ce qui évite de mélanger les laits de provenances diverses.

Quatre grands baes-balances sont répartis sur la plate-forme. Toute la machinerie est du reste disposée sur 4 échelons pour le traitement de 4 laits : 2 pour le lait naturel, 1 pour le lait écrémé, 1 pour la crème. De même qu'on trouve 4 machines de pesée, on trouvera 4 pasteurisateurs, et 4 séries de machines pour la mise en bouteilles : cette division du travail, ce "taylorisme ", pour employer le mot scientifique, est du reste l'un des caractères principaux de l'organication de la K. M.

Les quantités de lait fournies sont immédiatement reportées sur un registre. Une rapide analyse au goût et à l'odeur permet de recevoir ou de refuser le lait. Une prise d'échantillons pour les épreuves de la réductase et du lactofermentateur, ainsi que pour l'analyse de la M. G. au Gerber est également faite très soigneusement.

Du bac-balance, le lait est dirigé vers des appareils de nettoyage, sorte de centrifuges qui opèrent la séparation d'avec le lait de corps étrangers ou de fines poussières (1). A sa sortie du inettoyeur 》) le lait prend la direction du pasteurisateur, qui le porte à une température

(1) Le nettoyage du lait pour enfants est effectué avec un soin tout spécial. Ce lait est filtré à travers trois lits de graviers et de sable enfermés dans un appareil de forme cireu. laire qu'imagina dernièrement un ingénieur de la société. Journellement, on stérilise ces couches filtrantes à l'aide d'une lessive de soude et d'un jet de vápeur à degré très élevé. 
minima de $85^{\circ}$ dans le cas de fabrication de lait pasteurisé. Une vente de lait non pasteurisé qui atteint environ 3 à 4.000 litres de lait par jour a amıné pour une certaine quantité de lait la suppression de l'opération de pasteurisation.

La salle də réfrigération qui se trouve au deuxième étage, en dessous de la prósédente, reçoit alors par simple tuyauterie, sans pompe, le lait pasteurisé ou non pasteurisé pour le déverser à la partie supérieure des ráfrigáranłs qui l’amènent à une température de 2 à 3 degrés. D

Das réfrigérants, le lait passe dans de grands bacs assembleurs, à parois épaisses, mauvaises conductrices pour la chaleur et qui sont constituéss d'une paroi intérieure en cuivre, d'une paroi intermédiaire en liègə et d'une paroi interne. Neuf bacs construits sur ce modèle occupənt entièrement une salle accessible au seul employé chargé de la surveillanse de la température et de l'ésoulement du lait. Dans ces bacs mélangeurs, les laits et crèmes de provenances diverses se mélangent intimement et finissent par constituer après quelques brassages délicats effectués à la main avec un rateau mélangeur, des masses très homogènes, ce qui est indispensable avant la mise en bouteilles pour la vente au détail. De cette salle, située au premier étage, le lait coule au rezde-chaussée dans le hall de la mise en bouteilles.

Cette salle constitue à mon avis le point le plus intéressant de l'organisation de la laiterie de la K. M.

L'embouteillage et le capsulage, deux opérations consécutives, sont effectués lautomatiquement par quatre séries de machines très perfectionnés, correspondant aux quatre séries de l'outillage dont il a été question plus haut.

Las bouteilles arrivent par des voies automatiques, sortes de tapis roulants constitués par des chaînes sans fin, de la salle de nettoyage et d'aseptie, où des appareils de nettoyage à grand rendement, toujours au nombre de quatre, leur ont fait subir un traitement prolongé, depuis le moment où elles sont débarquées des voitures de livraison, au retour des tournées quotidiennes.

Ces appareils de nettoyage et de lavage intensif, qui peuvent traiter de 3.500 à 5.500 bouteilles à l'heure, bouteilles de tous calibres, depuis le litre jusqu'au dixième de litre prennent les bouteilles et leur font subir des nettoyages successifs sans brassages : d'abord à l'eau pure à température normale, la température monte progressivement jusqu'à $75^{\circ}$. A $40^{\circ}$, le lavage à l'eau de soude commence. Puis la température diminue jusqu'à un dernier lavage à l'eau pure. Chaque bouteille est lavée par 21 jets successifs, ce qui permet un nettoyage des plus satisfaisants.

A la sortie des laveurs, les bouteilles sont mirées à la lumière électrique, et repassent au lavage, au cas où celui-ci n'aurait pas été suffisant, ce qui est exceptionnel. Ce poste de "mireur ", assuré par des femmes, est des plus fatigants; les relais ont lieu toutes les heures. 
Les " embouteilleuses " prennent une à une les bouteilles et leur font décrire une circonférence, temps pendant lequel la bouteille se remplit jusqu'au goulot, à l'aide d'un dispositif très ingénieux de création récente. Le lait arrive des bacs mélangeurs à la partie supérieure des « embouteilleuses ", et se répand dans les récipients, par une petite canalisation verticale qui arrive à environ 2 ou 3 centimètres du fond de la bouteille pour éviter la production de mousse et une agitation du lait, toujours néfaste à la qualité de ce dernier. A sa sortie, la bouteille est saisie par la " capsuleuse " qui la bouche hermétiquement, avec une capsule en carton (capsule en métal pour le lait pour enfants), capsule dont la couleur et les inscriptions correspondent à la nature du lait contenu.

Une ouvrière colle l'étiquette appropriée et la bouteille s'en va toujours automatiquement vers la salle d'emballage et de départ.

Les embouteilleuses passent 3.500 bouteilles à l'heure, 'ce qui facilite beaucoup le travail, quand on considère que la K. M. livre 72.000 bonteilles par jour.

Un dispositif rapide permet d'équiper indifféremment les machines pour la mise en bouteilles du lait en récipients de 1 litre, $1 / 2$ litre, $1 / 4$ de litre, et même $1 / 10$ de litre, ces derniers récipients étant employés pour la crème.

Les bouteilles sont par la suite mises en caisses, qui ont également subi un nettoyage intégral à l'eau et à la vapeur.

Ces caisses sont ensuite entreposées dans une salle réfrigérée, où elles attendent de 1 heure de l'après-midi à 11 heures $1 / 2$ du soir, époque où se fait le traitement, jusqu'au lendemain matin de bonne heure, le départ pour la livraison (à partir de 4 heures du matin).

Cette livraison se fait par 90 voitures : 85 voitures à 2 chevaux, au mode typique bien connu à Copenhague, et 5 camions. Chaque conducteur a 2 assistants qui l'aident au chargement et au déchargement comme à la livraison des produits ; c'est qu'en effet la Compagnie se charge de porter à domicile la plus petite quantité de lait demandée : au $5^{\mathrm{e}}$ étage même $1 / 10$ de litre.

Une pièce spéciale sert au traitement des bidons vides, une fois le lait envoyé dans les bacs-balances. Ces bidons descendent par un tapis roulant sur un plan incliné vers une sorte d'immense autoclave, d'un modèle danois récemment conçu, où ils sont passés successivement à l'eau normale, à l'eau de soude, à la vapeur d'eau et finalement à la vapeur sèche, à raison de 350 bidons par heure; 1.200 bidons sont ainsi traités en moyenne par jour.

Après ce lavage, ils sont ramenés automatiquement aux wagons ou camions.

Une buanderie moderne lave et entretient les vêtements blancs donnés à tout le personnel : 2 fois par semaine, ces vêtements sont changés. 
A côté du lait naturel pasteurisé ${ }^{1}$ ou non (pasteuriset malk ou sod maslk), la sosiété livre à la consommation :

Io Environ 3 à 4.000 litres de lait pour enfants, Bornemaelk. Le lait subit un traitement analogue à celui du lait ordinaire non pasteurisé, mais comme il a été dit, dans une installation indépendante. Le nettoyage est spázialement surveillé et les récipients sont constitués par des bouteilles à goulot étroit, ce qui assure une fermeture plus parfaite. De plus ce lait est enrichi en crème ( $5 \%$ de matière grasse).

$2^{\circ}$ Le lait de Jersey : ou Jerseymaelk, produit par des vaches de la race de Jersey; c'est un lait très riche en matière grasse, 5 à $6 \%$. La capsule de fermeture doit porter la date de mise en bouteilles.

1.300 litres sont vendus par jour.

$3^{\circ}$ Les crèmes de plusieurs catégories suivant la teneur en matière grasse. On distingue :

a) crème fraîche à $30 \%$ Piske Flode ( teneur en M. G.

b) $\triangle$ No 2 flode $13 \% \quad$ inscrite

c) " " No 3 flode $\mathrm{N}^{\circ} 3$ à $9 \%$ sur capsules

Environ 2.000 litres de crème sont livrés à la consommation journalière.

$4^{\circ}$ Le lait écrémé avec le babeurre. Past. Kaernemaell. Il en est fait une assez grande consommation (4.500 litres par jour).

Ce Kaernemaelk est obtenu par un mélange de babeurre, de lait écrémé et d'un peu de crème mélangés dans des bacs où le tout séjourne 18 heures, à une température de $20^{\circ}$ et où l'on lui fait subir une fermentation assez accusée à l'aide d'un ensemencement avec des levures : ce Kaernemaelk a un goût acide très prononcé.

$5^{\circ}$ Le beurre est fabriqué dans la salle spéciale de beurrerie à l'aide de 3 grandes barattes-malaxeurs.

Environ 700 kilos de beurre sont faits quotidiennement. Cette quantité est insuffisante pour la demande des clients, aussi la K. M. F. achète-t-elle du beurre en gros pour le livrer à la consommation au détail.

Le beurre est livré en kilos, demi-kilos, ou demi-livres, soit en petites mottes entourées de papier sulfurisé protecteur, soit en pots de grés, d'un modèle très pratique.

Le client reçoit à domicile son beurre en même temps que son lait.

Un vaste laboratoire permet d'effectuer des analyses nombreuses et rapides des différents laits traités.

Le personnel se compose au total d'environ 500 ouvriers et employés.

Le lait est payé au producteur d'après sa teneur en matière grasse ; cette teneur est révélée par l'analyse au Gerber.

(1) Pour le lait pasteurisé, la eapsule de fermeture doit indiquer la date de la mise en bouteilles. 
Le barème des prix est établi chaque semaine d'après le prix du beurre établi par la commission qui décide chaque jeudi à Copenhague de la " cotation " du smör.

Actuellement le lait est payé aux producteurs une moyenne de 20 öres le litre (février 1928).

Las prix de vente sont à la même époque de ;

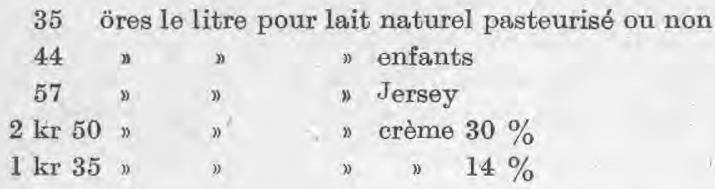

La vente de ces divers produits est sensiblement constante en quantité toute l'année. Elle accuse un accroissement cependant vers la saison des fraises, en juillet; ce fruit est consommé en grosses quantités en tous endroits et à toute heure, au Danemark, et il est largement arrosé de lait ou de crème : cet accroissement atteint environ 5.000 litres de lait et 1.000 litres de crème.

La grosse difficulté qui surgit en France, pour l'obtention d'un lait au-dessus de tout éloge, d'un lait "pur de vaches saines " analogue au lait danois, est d'ordre économique : la surveillance des étables, et le soin tout spécial apporté à une alimentation rationnelle entraînent de gros frais pour le cultivateur, et celui-ci a le droit d'exiger pour cette production soignée un prix suffisamment rémunérateur.

Les conslusions et les enseignements à tirer de cet exposé semblent pouvoir se résumer dans les quelques lignes ci-dessous :

$1^{\circ}$ Parfaite organisation de la production d'un lait véritablement hygiénique ;

$2^{\circ}$ Contrôle serré, exercé par le service privé de la société, travaillant, en coopération avec le service officiel du contrôle de l'Etat, à donner au consommateur une garantie formelle, au-dessus de tout soupçon, pour le produit acheté.

$3^{\circ}$ Equipement des plus rationnels et des plus modernes permettant de travailler rapidement et dans les meilleures conditions économiques, à livrer à la consommation de grosses quantités de laits variés.

Tel est l'idéal auquel viennent d'aboutir par de longs efforts, grâce à un esprit toujours tourné vers le progrès, ces deux grandes sociétés laitières, la D. M. C. et la K. M. F. aux emblèmes significatifs, pour I'une, l'ours polaire sur la banquise, élevant entre ses pattes le bidon de lait, et pour l'autre, les deux fleurs de trèfle, symboles très vivants d'un lait supérieur obtenu par une alimentation soignée des vaches laitières (1).

(1) La * Maypole Cy grande société anglaise vient de construire près de Londres, une laiterie sur le modèle de celle appartenant à la K. M. F. 


\title{
$2^{\circ}$ La Société Enigheden.
}

C'est l'une des trois grandes laiteries commerciales de Copenhague, approvisionnant la capitale en lait de consommation. Son organisation ressemble beaucoup a celle de la Danske Maelke Compagni, qui a été longuement étudiée, aussi il ne sera dit que quelques mots de Mejeriet Enigheden.

Créée en 1898 sur l'initiative socialiste, dans le but de procurer aux ouvriers un lait de toute première qualité à un prix aussi modique que possible, la Société Enigheden, qui a subi des transformations successives pour se moderniser, occupe un vaste emplacement à Alexandravej, en bordure de Copenhague.

Elle traite journellement de 50 à 60.000 kilos de lait, fourni par une cinquantaine d'exploitations.

A titre de particularités, il faut signaler la présence d'un homogénéisateur à 4 cylindres horizontaux de construction danoise, et des bacs assembleurs de 5 à 6.000 kilos de lait conservant le lait à une température constante de 5 à $6^{\circ}$, et pourvus d'agitateurs ingénieux destinés à égaliser le lait et à empêcher la crème de monter; sans la présence de ces agitateurs le lait situé au fond du bac serait moins riche que le lait de la partie supérieure.

La caractéristique de la Société Enigheden réside dans le soin tout particulier apporté au traitement du lait pour enfants, le "Bornemaelk».

Ce lait est produit à Lautrupgaard, dans une ferme appartenant à la société, et où tout est mis en œuvre pour recueillir un lait aussi pur que possible : un troupeau d'élite de la race rouge, soumis à la surveillance permanente d'un vétérinaire, et recevant une alimentation substantielle fournit le lait pour enfants.

Les soins apportés à la traite sont très particuliers (voir la réglementation du lait pour enfants) et le personnel est soumis à un cortrôle médical constant, par les soins d'un docteur attaché à la maison.

Environ 2.000 kilos de lait pour enfants sont ainsi distribués tous les jours dans la capitale après traitement à la laiterie d'Alexandravej.

\section{LA MÉTHODE SYNTHÉTIQUE DANS L'ÉTUDE DU LAIT LE LAIT AU POINT DE VUE COLLOIDAL RECHERCHES SUR LE MÉCANISME DE L'ACTION DE LA PRÉSURE}

par Ch. PORCHER,
Docteur ès sciences physiques

\author{
(Suite) \\ CHAPITRE $V$
}

\section{LES COMPOSÉS DE LA CASÉINE}

La caséine, ainsi qu'il résulte de la préparation que nous en avons donnée dans le chapitre précédent et de diverses considérations dans lesquelles nous étions entré antérieurement, est bien une entité chimique 